\title{
Accretion-ejection instability and QPO in black-hole binaries
}

\section{Relativistic effects}

\author{
P. Varnière, J. Rodriguez, and M. Tagger
}

DSM/DAPNIA/Service d'Astrophysique (CNRS URA 2052), CEA Saclay, 91191 Gif-sur-Yvette, France

Received 23 May 2000 / Accepted 25 February 2002

\begin{abstract}
The Accretion-Ejection Instability has been proposed to explain the low frequency Quasi-Periodic Oscillation (QPO) observed in low-mass X-Ray binaries, in particular Black-Hole candidates. Its frequency, typically a fraction of the Keplerian frequency at the disk inner radius, is exactly in the range indicated by observations. The variations of the frequency with the disk inner radius (extracted from spectral fits of the X-ray emission) might thus be a useful test. In this paper we discuss how changes in the rotation curve, due to relativistic effects when the disk approaches the central object, affect the physics of the instability, and thus this frequency-inner radius relation. We find that the relationship between the frequency of the mode and the Keplerian frequency at the inner disk radius $\left(r_{\text {int }}\right)$ departs from the one obtained in a Keplerian disk, when $r_{\text {int }}$ approaches the last stable orbit. This might agree with the recently published results, showing a discrepancy between the behavior of the QPO in the micro quasar GRO J1655-40, compared to other sources such as XTE J1550-564 and GRS 1915+105. In a companion paper (Rodriguez et al. 2002, hereafter Paper I) we have presented detailed observational results for GRO J1655-40 and GRS 1915+105. We show how the opposite correlations found in these sources between the disk color radius (assumed to be close to its inner radius) and the QPO frequency could indeed be explained by our theoretical result.
\end{abstract}

Key words. accretion, accretion disks - instabilities - MHD - waves - galaxies: jets

\section{Introduction}

This is the second of two papers where we compare the properties of the Accretion-Ejection Instability (AEI), recently found to occur in the inner region of disks with a moderate magnetic field (Tagger \& Pellat 1999, hereafter TP99), with the low frequency Quasi-Periodic Oscillation (QPO) observed in galactic Black-Hole binaries.

The AEI is a spiral instability, driven by magnetic stresses, of disks threaded by a magnetic field of moderate (i.e. near equipartition) amplitude. It belongs to the same family as galactic spirals, driven by self-gravity (see Binney \& Tremaine 1987 and references therein) or the Papaloizou-Pringle instability (Papaloizou \& Pringle 1985), driven only by pressure forces. It is essentially the same spiral instability found by Tagger et al. (1990); this instability was quite weak and thus unlikely to be very efficient in an accretion disk, but it was shown in TP99 that a different physical process, the corotation resonance (analyzed as a coupling with a Rossby wave in the disk) could give it a more sizable growth rate. Rossby waves are familiar in planetary atmospheres, where their most

Send offprint requests to: P. Varnière,

e-mail: pvarni@discovery.saclay.cea.fr spectacular manifestation is the Great Red Spot of Jupiter. They propagate in flows with a gradient of vorticity, a gradient most often neglected in analytical studies of disks.

In TP99 it was at the same time recognized that this resonance offers in a magnetized disk a unique prospect, responsible for the name given to the instability: the AEI grows (and causes accretion) by extracting energy and angular momentum from the disk, and storing them in a Rossby vortex at its corotation radius (the radius where the wave rotates at the same velocity as the gas in the disk). If the disk has a low-density corona, this energy and momentum will be re-emitted as Alfvén waves traveling upward to this corona, where they might power a wind or a jet. Thus, and although only a limited computation of this effect was given in TP99 (a full computation will be presented in a forthcoming paper), the AEI provides a unique way of connecting accretion and ejection in the disk. This contrasts with other known disk instabilities, or with the hypothesis of a turbulent viscosity, which all result in a radial transport of energy and momentum, making a connection with MHD models of jets very difficult. These models show that the jet is very efficient at carrying away angular momentum from the disk, 
and a mechanism connecting radial and vertical transport of angular momentum is thus highly desirable (see however Casse \& Ferreira (2000) and references therein). It is very interesting to note ${ }^{1}$ that the conditions of instability are always fulfilled by these MHD models of jets, since they have a plasma $\beta \sim 1$, and the radial gradient, which must be positive, always has (as a consequence of the self-similar ansatz) the value $+1 / 2$.

In this paper we will first present a comparison between the observed properties of the low-frequency QPO and the behavior expected from the AEI, showing that this instability could provide a good explanation for the QPO. In this respect we will turn to an effect neglected in TP99. In that paper it was found, from exact numerical solutions, that the $m=1$ mode (i.e. 1-armed spiral) was often the most unstable, although it could not be predicted from an approximate WKB theory. This was confirmed in numerical simulations by Caunt \& Tagger (2001). Here we first show that relativistic effects, when the inner edge of the disk approaches the Black Hole, change this special status of the $m=1$ mode, and introduce a qualitative difference in its properties. Numerical solution of the linearized MHD equations, using a pseudo-Newtonian potential which mimics the relativistic corrections to the rotation curve in the disk, then shows that the relation between the mode frequency and the disk inner radius is changed by these effects. This is potentially important since spectral fits of the disks of X-ray binaries give a measure of the disk inner radius: thus the correlation between this radius and the QPO frequency can be directly tested.

As we were completing this work which had started from a purely theoretical argument, new observations (Sobczak et al. 2000, hereafter SMR) showed that this frequency-radius correlation is reversed in the microquasar GRO J1655-40 compared to other sources - in particular another microquasar, XTE J1550-564. We suggest that these observations might correspond to our theoretical results. In our previous paper (Rodriguez et al. 2002, hereafter Paper I) we have critically reassessed the analysis of GRO J1655-40 by SMR and confirmed its reverse frequency-radius correlation. We then turned to another microquasar, GRS 1915+105, because its high variability and the large number of available observations let us hope to explore the correlated variations of the disk radius and the QPO frequency over a much broader interval. The results for GRS 1915+105 showed the usual correlation, opposite to that of GRO J1655-40. In our final discussion we will show that these opposite behaviors might be explained by our theoretical result.

In a recent paper Psaltis \& Norman (2002) have presented a model for the low-frequency QPO. It is a model of random excitation of modes (analogous in a way to the excitation of solar modes), which are filtered at a transition radius assumed to exist in the disk. The QPO frequencies then lie at (or near) fundamental single-particle frequencies at this radius. In that case the low frequency

\footnotetext{
${ }^{1}$ J. Ferreira, private communication.
}

QPO we discuss here would be associated, as proposed by Stella \& Vietri (1999), with the nodal-precession frequency. The computation we present here is a more elaborate one (since it describes global hydrodynamical or MHD perturbations, rather than the individual motion of isolated particles or gas blobs), for one such type of mode (the spiral density wave), which is based on the epicyclic motion. It thus goes in the direction outlined by Psaltis (2002), moving from basic frequencies of motion to the hydrodynamical (in his case) or MHD (in our case) properties of flows in the disk. Our model is also different in that the modes are unstable: they grow spontaneously from the thermal noise and are expected (this has now been verified in numerical simulations by Caunt \& Tagger 2001) to form narrow and high-amplitude features, as galactic spirals do. This contrasts with the model of Psaltis and Norman, where a sharp transition radius must be assumed to exist in the disk to explain the excitation of the mode, and we have no a priori difficulty to explain the very high amplitude sometimes observed in the QPO.

\section{The Accretion-Ejection Instability}

\subsection{Basic properties}

For the sake of completeness we present here the main elements of physics underlying this instability mechanism. More details and discussions can be found in TP99.

This instability appears as a spiral density wave in an accretion disk threaded by a vertical (poloidal) magnetic field, of the order of equipartition with the gas thermal pressure. This part of the instability is essentially similar to the spiral density waves of galactic disks, but it is driven by magnetic stresses rather than self-gravity (Tagger et al. 1990). The action of differential rotation couples the spiral to a Rossby wave at its corotation radius (the radius where the wave rotates at the same velocity as the gas). This means that the spiral wave generates, at its corotation radius, a Rossby wave in which it stores the energy and angular momentum it extracts from the disk (thus causing accretion). It is this exchange of energy and angular momentum which makes the whole perturbation (density wave + Rossby wave) unstable, i.e. growing exponentially with time.

A standing wave pattern (called in this context a normal mode) can form in the following manner, also responsible for the standing spiral pattern in galaxies: consider a spiral wave propagating outward from the inner radius of the disk. As it approaches corotation it is reflected as another spiral wave, propagating inward; it is during this reflection that some wave energy is exchanged (by the action of differential rotation) with the Rossby wave, so that the reflected spiral has a higher amplitude than the original one. Now the reflected spiral, as it reaches the inner disk radius, is reflected again as an outgoing spiral. This forms an equivalent to an electromagnetic cavity: if the resulting outgoing spiral has the same phase as the initial one, the process will repeat itself, the whole 


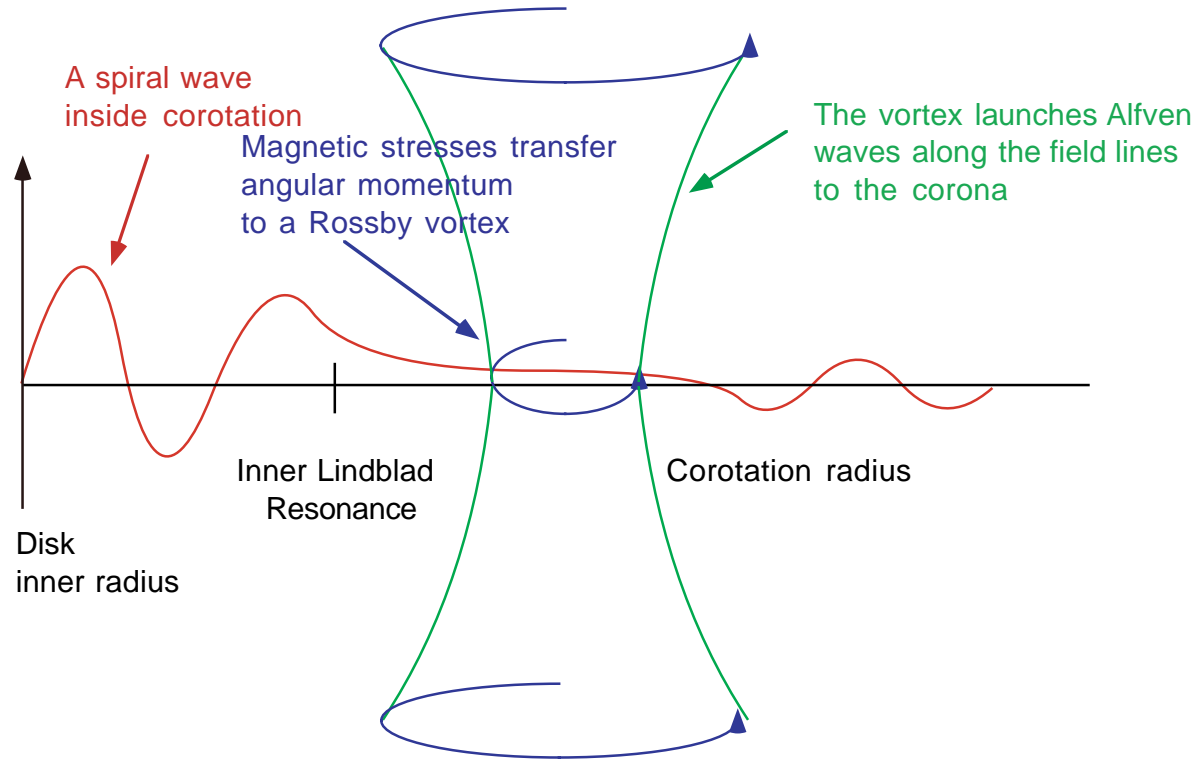

Fig. 1. The structure of the instability is described here schematically as a function of radius. It is formed of a standing spiral density wave in the inner part of the disk, coupled to a Rossby vortex it excites at its corotation radius. The Rossby vortex in turn generates Alfvén waves propagating toward the corona of the disk.

pattern being amplified at each reflection at corotation. As in an electromagnetic cavity, this integral phase condition selects a discrete set of frequencies, corresponding to $n_{\mathrm{r}}=0,1,2 \ldots$ nodes in the radial structure of the spiral wave pattern, known as normal modes of the system. In this process the Rossby wave becomes a standing vortex, rotating at the angular velocity of the pattern. In practice only the $n_{\mathrm{r}}=0$ mode, the most unstable one, will concern us here. Figure 1 illustrates the trajectory of the waves within the cavity, and the excitation of the Rossby wave.

This basic physical description was obtained in the simple model of a thin disk in vacuum, and numerical solutions were given in TP99. If now one takes into account a low density corona above the disk, it was shown in TP99 that the waves in the disk will generate an Alfvén wave transferring to the corona (where it might power a wind or an outflow) the energy and angular momentum extracted from the disk. The AEI thus appears as a very good candidate to connect accretion and ejection in the inner region of a magnetized disk. The computation of the Alfvén wave emission was done, in TP99, in a WKB approximation valid only away from the corotation region, where most of the emission should occur. A full computation (Varnière \& Tagger 2002) will be given in a forthcoming publication.

\subsection{The Inner Lindblad Resonance}

The propagation properties of the waves determine the mode frequency, and thus the location of its corotation radius. It was shown in TP99 that the point where the wave is reflected, near its corotation radius, is in fact the Inner Lindblad Resonance (ILR), where the Dopplershifted wave frequency

$\tilde{\omega}(r)=\omega-m \Omega(r)$ where $\omega$ is the wave frequency, $m$ is the azimuthal wavenumber (i.e. the number of spiral arms) and $\Omega$ is the rotation (orbital) frequency, is equal to the opposite of the epicyclic frequency $\kappa$, given by

$\kappa^{2}=4 \Omega^{2}+2 \Omega \Omega^{\prime} r$

where the prime notes the radial derivative. $\kappa$ is the restoring frequency which appears in the motion of individual particles (stars in the galactic context, fluid elements here), initially on a circular orbit, and to which a radial perturbation is given. When describing fluid motions, $\kappa^{2}$ appears as a restoring force, which supplements pressure and other forces (gravity in the galactic context, magnetic stresses here).

The wave propagates in the region where $\tilde{\omega}^{2}-\kappa^{2}>0$, i.e. in the radial interval between the inner disk radius $r_{\text {int }}$ and the ILR radius. This is where a cavity is formed, and where a standing pattern results from the combination of the ingoing and outgoing waves, as shown in Fig. 1. The manner in which waves are reflected at the Inner Lindblad Resonance and at the inner disk edge has been discussed in details in TP99.

When studied in a WKB approximation for the radial structure of the wave in a Keplerian disk, the wave is found to be evanescent (non-propagating) in the region between the ILR and the corotation radius. It was also shown in TP99 that in this WKB approximation this results in a mode frequency close to

$\omega \lesssim(m-1) \Omega_{\text {int }}$

where $\Omega_{\text {int }}$ is the rotation frequency at the inner radius $r_{\text {int }}$, and $m$ is the azimuthal wavenumber (i.e. the number of spiral arms). From this WKB approximation the $m=1$ mode seems to be excluded, since it does not have an ILR because $\kappa=\Omega$ in Keplerian rotation around a 
central mass (so that $\omega-\Omega=-\kappa$ would imply $\omega=0$ ). However the WKB analysis is only marginally valid, and should be used only as a guide. In practice it was shown in TP99 by exact numerical solution of the problem that the $m=1$ mode does exist, and is often the most unstable. This was to be expected, since the physics of Rossby waves usually selects large wavelengths (e.g. the Great Red Spot of Jupiter), and since the efficiency of the corotation resonance (the coupling between spiral and Rossby waves) is found to scale roughly as $m^{-1}$. Thus $m>1$ modes have their corotation radius very close to $r_{\text {int }}$, whereas the $m=1$ typically has a frequency of the order of $.1-.3 \Omega_{\text {int }}$, depending on various disk parameters; this corresponds to a corotation radius at a few times $r_{\text {int }}$. As discussed in the next section, this frequency range is the observed one for the low frequency QPO.

Relativistic effects may be expected to make a strong qualitative difference here: indeed the last stable orbit at $r_{\text {LSO }}$ is defined as the orbit where, because of these effects, the epicyclic frequency $\kappa$ vanishes, whereas $\kappa=\Omega$ in a Newtonian, Keplerian disk. One must remember that $\kappa$ corresponds to the restoring force experienced by a particle moving away from a circular orbit. For $r<r_{\mathrm{LSO}}, \kappa$ is imaginary so that the radial motion of orbiting particles is unstable and they will rapidly spiral toward the central object. The vanishing of $\kappa$ at $r_{\text {LSO }}$ (rather than $\kappa=\Omega$ in a Newtonian, Keplerian disk) thus introduces the possibility for an $m=1$ mode to have an ILR, if the disk comes close enough to $r_{\mathrm{LSO}}$; we expect (and will indeed find below) this to affect the properties of the $m=1$ mode.

In the low state of black-hole binaries, $r_{\text {int }}$ is usually found to be a few times $r_{\mathrm{LSO}}$, although we do not know what can exist between $r_{\text {int }}$ and the black hole: an ADAF might be a possibility, but one might also think of the force-free magnetic structure necessary to contain the vertical magnetic flux threading the black hole, according to the Blandford-Znajek (1977) mechanism. However certain observations, which have been discussed in detail in Paper I, show that sometimes, during its variation, $r_{\text {int }}$ has a lower bound which probably marks the position of $r_{\text {LSO }}$. This has prompted us to study the effect of the existence of an ILR on the frequency and growth rate of the $m=1$ mode. We will then compare the theoretical result with the observational one of Paper I.

\section{AEI and QPO properties}

Before taking into account the pseudo-Newtonian effects we will concentrate on how the AEI can be associated with the QPO. In this view we will first make a brief summary of the characteristics of QPOs.

Quasi-Periodic Oscillations have been widely observed in many X-ray binaries, whose compact object is either a neutron star or a black-hole candidate. They are commonly considered to originate in the disk, either at its inner boundary (in particular the $\mathrm{kHz}$ QPO in neutronstar binaries) or beyond it. Among black-hole binaries, the low-frequency QPO (of the order of one to a few $\mathrm{Hz}$ ) has drawn particular attention, because it seems to convey important information on the physics of the inner region of the accretion disk and of the corona.

In particular in the micro-quasar GRS 1915+105, Swank et al. (1997) and Markwardt et al. (1999) (hereafter SM97 for both of these references), who dubbed it "ubiquitous" since it seems to be always present in the low-hard state of the source, have shown that its frequency varies with the evolution of the disk, during its low and hard state. Indeed a correlation can be found, in two different manners:

- The first correlation seems to relate the QPO frequency with the color radius of the disk. This radius is obtained from the model (multicolor blackbody+power-law tail) commonly used to fit the X-ray spectrum of the source. It is considered as a measure of the inner radius of the disk, even though the exact relation between them is not well known. We will discuss in this paper only in terms of the disk inner radius, but will return below to the inner radius - color radius relation.

In the case of GRS $1915+105$, during a typical $30 \mathrm{~min}$ cycle between a high/soft and a low/hard state, SM97 show that the QPO appears only during the low state, and that its frequency varies with the disk parameters. If the QPO frequency is converted into an equivalent Keplerian radius in the disk, assuming a reasonable mass for the black hole, this radius would be of the order of a few times the observed color radius. Furthermore, the QPO frequency seems very well correlated with the color radius. Indeed, in a detailed discussion of the QPO in various states of the source, Muno et al. do show such a correlation. In particular, during the 30 min cycles of GRS $1915+105$, the QPO frequency can be seen to decrease with increasing radius. Both studies conclude that the QPO, although it affects more strongly the power-law tail (coronal emission), seems to have its origin in the disk.

- A second correlation, found by Psaltis et al. (1999a) (hereafter P99), is more fragile at the present time since it relies on very few data for black-hole binaries. Recent results by Nowak (2000), finding three broad peaks in the spectra of black-hole binaries, might indicate that there is more to be discovered in that direction. The result of Psaltis et al. is very interesting because it fits, in a very different manner, with the previous one: they show that, in a large variety of $\mathrm{X}$-ray binaries, there seems to be a correlation between a high and a low frequency QPO: the low-frequency QPO involved in the correlation (which is the QPO discussed by SM97 and by SMR) is observed in many sources, whose compact object is either a neutron star or a black hole. Its frequency ranges from about 1 to a few tens of $\mathrm{Hz}$. The high-frequency QPO in the correlation differs between neutron stars and black hole binaries: in neutron stars it is the lower of the pair of so-called " $\mathrm{kHz}$ QPO", believed to originate at the 
inner disk radius; the higher-frequency QPO of the pair would correspond to the Keplerian rotation frequency at this radius, while the lower-frequency one might be a beat wave between this and the rotation frequency of the neutron star (Miller et al. 1998), or the periastron precession frequency (Stella \& Vietri 1999). We will not discuss these models here (see e.g. Psaltis et al. $1999 b)$, but only retain that the upper frequency, in the correlation of Psaltis et al., is close to the Keplerian rotation frequency near the inner edge of the disk. In black-hole binaries, the high-frequency QPO considered in the correlation is seen as a broad feature at a few tens of Hz. This correlation can thus be seen as relating the frequency of the low-frequency QPO and the inner radius of the disk.

The correlation found by Psaltis et al. is such that the ratio between the two frequencies is about 11, corresponding to a ratio $\sim 5$ between the corresponding radii if one considers them as Keplerian frequencies. This is comparable to the ratio found by SM97.

The exact relation between the color radius given by the spectral fit and the true inner radius of the disk is not well known and an open debate among observers, since it involves a number of corrections which depend on assumptions about the disk and coronal structures. Different works explore this relation, such as Shimura \& Takahara (1995) in the case of a Schwarzschild black-hole, or Merloni et al. (1999), but a general agreement on the correction to be made is still lacking. In this work we will simply identify $r_{\text {col }}$ and $r_{\text {int }}$, and will discuss in our conclusions how this affects our results.

We consider the AEI as a very good candidate to explain the QPO because of a number of characteristics:

- Its frequency, for the one-armed spiral ( $m=1$, where $m$ is the azimuthal wavenumber) is a fraction of the Keplerian frequency at the inner radius, fully compatible with the observation. This would explain both the absolute value of the QPO frequency and its correlation with the inner disk radius. We focus on the $m=1$ mode because the theory shows it (or the $m=2$, depending on disk parameters) to be most unstable, and because recent numerical simulations (Caunt \& Tagger 2001) show that the disk evolution most often leads it to dominate. The $m=2$ would have a higher frequency than the low-frequency QPO we discuss here.

- As in galaxies, its physics should make it form longlived, standing patterns, so that it should appear as what is called in the galactic context a quasi-stationary spiral structure - as confirmed by the numerical simulations. This would explain the long life of the QPO (or rather its long correlation time, measured by the width of the QPO), as observed in particular during the hard and steady state of the source.

- These properties would probably be shared by any spiral mode, driven by other instability mechanisms (e.g. the Papaloizou-Pringle instability, although in that case the growth rate is weaker and concerns only high- $m$ modes). However the AEI has the additional property of emitting vertically, as the Poynting flux of an Alfvén wave, the energy and angular momentum extracted from the disk (whence the name we have given to this instability); this makes it a realistic explanation, both for the compact jet observed in these sources (e.g. Dhawan et al. 2000) and for the strong modulation of the coronal emission with the QPO.

Based on these considerations, we have discussed (Tagger 1999) how, if the QPO is indeed identified with the AEI, this could lead to a possible scenario for the $30 \mathrm{~min}$ cycles of GRS $1915+105$. In this scenario accretion in the inner region of the disk, and the observed cycles, would be controlled by the accumulation of poloidal magnetic flux in the disk.

These points may be considered only as favorable indications - though better than for other models of QPO, where most often only basic frequencies of motion are identified, without a mechanism of wave excitation. The basic physics and other theoretical expectations (e.g. the formation of a long-lived, quasi-stationary spiral pattern) have been confirmed by numerical simulations (Caunt \& Tagger 2001). This has led us to look for more distinctive observational signatures of the AEI.

\section{Relativistic effects}

A fully relativistic description of the instability is a formidable challenge we will not attempt here. Instead we use existing models of pseudo-Newtonian potentials, devised to mimic the behavior of the rotation curve (and in particular the vanishing of $\kappa$ at $r_{\mathrm{LSO}}$ ) in the relativistic part of the disk. This is enough to check the effect, on the $m=1$ mode, of the apparition of an Inner Lindblad Resonance in the disk.

\subsection{The pseudo-Newtonian potential}

We use the pseudo-Newtonian potential given by Nowak \& Wagoner (1992):

$\Phi=-\frac{G M}{r}\left(1-3 \frac{G M}{r c^{2}}+12\left(\frac{G M}{r c^{2}}\right)^{2}\right)$.

This is a second order approximation of the Schwarzschild metrics, so that it neglects the effect of the spin of the compact object. Although the angular momentum arising from the spin of the black hole might change the radius of the last stable orbit by a factor up to six for extreme spin, the qualitative effect of the presence of an ILR for the $m=1$ mode would remain.

From this potential one can compute the orbital and epicyclic frequencies:

$r \Omega^{2}=\frac{\partial \Phi}{\partial r}$
$\kappa^{2}=r \frac{\partial}{\partial r} \frac{1}{r} \frac{\partial \Phi}{\partial r}$ 


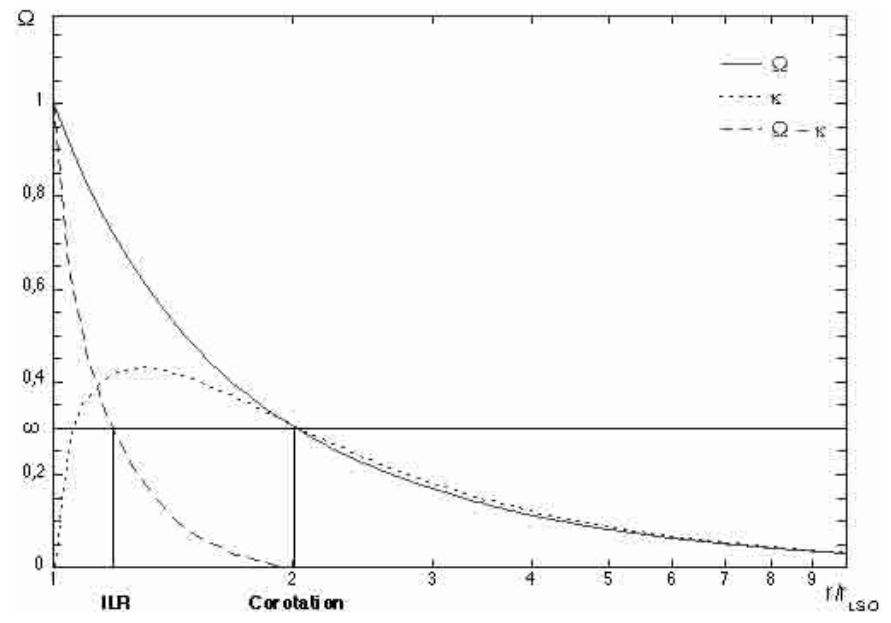

Fig. 2. Rotation curves in a pseudo-Newtonian potential, showing the frequencies $\Omega$ (solid), $\kappa$ (dots) and $\Omega-\kappa$ (dashed), normalized to the orbital frequency at the Last Stable Orbit $\left(r=r_{\text {LSO }}\right)$. The line at $\omega=.3$ shows for example that a mode of frequency $\omega=.3 \Omega\left(r_{\mathrm{LSO}}\right)$ has a corotation and ILR respectively near $r \simeq 2 r_{\mathrm{LSO}}$ and $r \simeq 1.2 r_{\mathrm{LSO}}$, if these radii are within the disk (i.e. if the inner radius $r_{\text {int }}$ is small enough).

so that the last stable orbit is at

$r_{\mathrm{LSO}}=6 \frac{G M}{c^{2}}$

and $\Omega$ and $\kappa$ can be rewritten as:

$$
\begin{aligned}
& \Omega=\sqrt{G M} r^{-\frac{3}{2}}\left(1-\frac{r_{\mathrm{LSO}}}{r}+\left(\frac{r_{\mathrm{LSO}}}{r}\right)^{2}\right)^{\frac{1}{2}} \\
& \kappa=\sqrt{G M} r^{-\frac{3}{2}}\left(1-\left(\frac{r_{\mathrm{LSO}}}{r}\right)^{2}\right)^{\frac{1}{2}} .
\end{aligned}
$$

Figure 2 shows the resulting rotation curve, and the function $\Omega-\kappa$. It shows how relativistic effects (or a pseudoNewtonian potential) allow $\kappa$ to decrease near $r_{\text {LSO }}$, so that the $m=1$ mode can have an ILR when $r_{\text {int }}$ is close enough to $r_{\text {LSO }}$.

For the numerical solution, we have also used as a cross-check the pseudo-Newtonian approximation of Paczynski \& Witta (1980):

$\Phi=-\frac{G M}{r-r_{\mathrm{g}}}$

where $r_{\mathrm{g}}=2 G M / c^{2}$ is the Schwarzschild radius. This Padé approximation (rather than the series expansion above) gives essentially the same results, confirming our expectation that the results depend mostly on the qualitative difference introduced by the existence of an ILR, rather than on the detailed rotation curve; we will present here only results using Eq. (1).

\section{Numerical results}

\subsection{Numerical method}

We use the same method of solution as in TP99, and the same setup: the disk is supposed to be infinitely thin and embedded in a vacuum. It is threaded by a vertical magnetic field $\boldsymbol{B}=B_{0}(r) \boldsymbol{e}_{z}$. For the sake of completeness, we repeat here the system of linearized MHD equations solved:

$$
\begin{aligned}
& -i \tilde{\omega} U-2 \Omega V=-c_{\mathrm{s}}^{2} \frac{\partial h}{\partial s}-2 \frac{B_{0}}{\Sigma} \frac{\partial \Phi_{\mathrm{M}}}{\partial s} \\
& -i \tilde{\omega} V+W U=-i m c_{\mathrm{s}}^{2} h-2 i m \frac{B_{0}}{\Sigma} \Phi_{\mathrm{M}} \\
& -i \tilde{\omega} r^{2} \sigma=-\frac{\partial}{\partial s}(\Sigma U)-i m \Sigma V \\
& -i \tilde{\omega} r^{2} B_{z}^{\mathrm{D}}=-\frac{\partial}{\partial s}\left(B_{0} U\right)-i m B_{0} V
\end{aligned}
$$

where $s=\ln r, U=r v_{\mathrm{r}}, V=r v_{\vartheta}, \Sigma$ and $\sigma$ are the equilibrium and perturbed surface densities, $h=\sigma / \Sigma$, $W=\kappa^{2} / 2 \Omega$ is the vorticity in the equilibrium flow, $B_{0}$ and $B_{z}^{\mathrm{D}}$ are the equilibrium and perturbed vertical magnetic fields at the surface of the disk, and $\Phi_{\mathrm{M}}$ is a magnetic potential obtained from:

$\nabla^{2} \Phi_{\mathrm{M}}=-2 B_{z}^{1} \delta(z)$

which is solved using the Poisson kernel commonly used in self-gravitating disks. In other terms, the magnetic field is described above the disk (in vacuum, with no currents) by a magnetic potential, whose source is the currents in the disk. For simplicity, since pressure plays very little role in the instability, we have assumed an isothermal equation of state.

The presence of $\Phi_{M}$ makes this an integro-differential system, corresponding to the long-range action of magnetic stresses (in contrast with the local action of pressure stresses between neighboring fluid elements). This system is solved by projecting it on a radial grid, evenly spaced in the variable $s=\ln r$. The solution then reduces to finding the eigenvalues and eigenvectors of a matrix: each eigenvalue is a frequency $\omega$ and the corresponding eigenvector gives the radial structure of the perturbed velocity, density and magnetic field. A discrete set of eigenvalues, corresponding to the modes with $n=0,1,2 \ldots$ nodes in their radial structure, is then easily identified, as discussed in TP99 (the other eigenvalues come from the discretization of the problem on a numerical grid). The boundary condition of an outgoing wave at large radius (i.e. no influx of information from the outer ranges of the disk) is implemented, as in TP99, by solving on an axis slightly tilted in the complex- $s$ plane.

The only change here is that we use in Eqs. (5)-(8) the rotation curve described above, rather than the Newtonian one used in TP99. We introduce the parameter

$\xi=r_{\text {int }} / r_{\text {LSO }}$

At large $\xi$ the whole disk is very close to the Newtonian rotation curve, and we recover the results of TP99. When $\xi$ becomes close to 1 , i.e. when the disk inner radius approaches the last stable orbit, relativistic effects on the rotation curve start to play and the $m=1$ mode can have an ILR. In that case, between $r_{\text {int }}$ and the ILR, the 
wave propagates (in a WKB sense). Let us compare this with the behavior of a simple oscillator, described by the equation:

$$
\frac{\mathrm{d}^{2}}{\mathrm{~d} x^{2}} \Psi+M \Psi=0
$$

(this comparison is quite relevant since, where the WKB approximation applies, the system of Eqs. (5)-(9) reduces to a system of this form).

In the propagation zone (i.e. for us between $r_{\text {int }}$ and the ILR) $M$ is positive, so that $\Psi$ oscillates. On the other hand in the "forbidden band" (for us, between the ILR and corotation, or between $r_{\text {int }}$ and corotation if no ILR is present) $M$ is negative and the mode has an exponential behavior ${ }^{2}$.

Thus the change in the behavior of the solutions between $r_{\text {int }}$ and the ILR will change the integral phase condition, which determines the mode frequency. This is true even though, as mentioned in the introduction, the WKB approximation is not really valid, resulting in the very fact that the $m=1$ mode exists even in a Newtonian potential. Our first goal is to quantify these effects.

\subsection{Numerical results}

The computation uses two more parameters: the magnitude of the magnetic field, and its radial profile. The field is measured by the parameter $\beta=2 \mu_{0} p / B^{2}$, i.e. the ratio of the thermal and magnetic pressures. As discussed in TP99, our instability occurs when $\beta$ is of the order of 1 or lower (whereas the magneto-rotational instability (Balbus \& Hawley 1991; Chandrasekhar 1960; Velikhov 1959) occurs only for $\beta>1$ ). We will present here only results with $\beta=.5$, and complement them with results at $\beta=1$ for comparison.

In the same manner, we use as in TP99 a flat radial density profile, and a magnetic field profile varying only over a limited radial range, around the corotation radius: it was shown in TP99 that the amplification of the mode depends only on the local gradient, at the corotation radius, of the quantity $\kappa^{2} \Sigma / 2 \Omega B_{0}^{2}$, and these somewhat artificial profiles allow us an easy solution, by limiting the global variation of equilibrium quantities across the numerical grid. The real part of the frequency depends of course on the global profiles. Here we want to consider only the effect of the relativistic modifications of the rotation curve. Thus we will stick to these simple radial profiles. We will report in a future publication results obtained

\footnotetext{
${ }^{2}$ Note however that, as described by Tagger et al. (1990), in our problem the behavior of $\Phi_{\mathrm{M}}$ in the forbidden band is algebraic rather than exponential; this is due to the long-range action of magnetic stresses, i.e. to the $r^{-m-1}$ dependence of an $m$-polar field. It allows waves in the inner cavity to couple quite efficiently, across the forbidden band, to the outgoing wave and to the Rossby vortex. This results in a stronger instability than the Papaloizou-Pringle one of unmagnetized disks, because in that case pressure forces result in a classical exponential decay in the forbidden band.
}

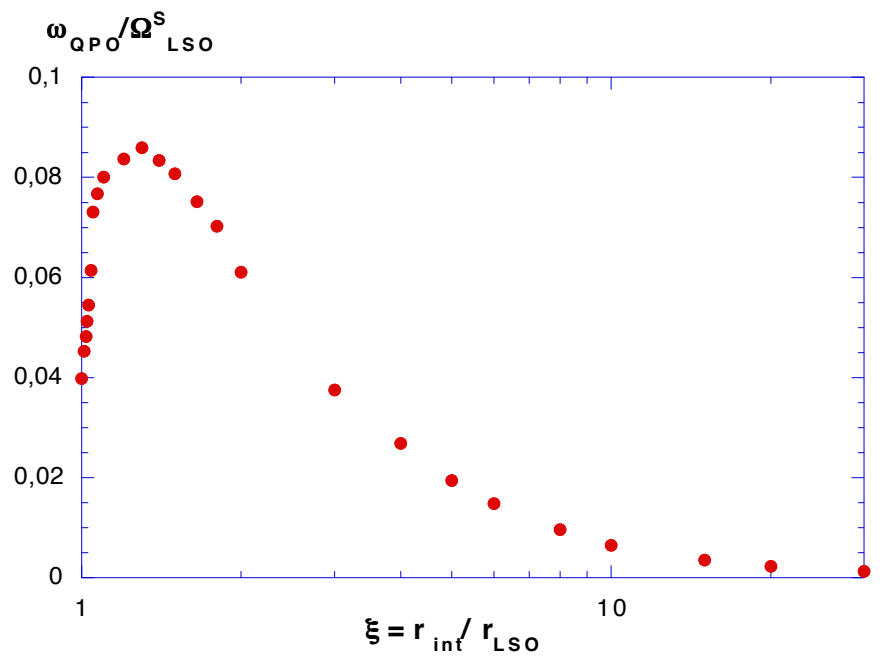

Fig. 3. Frequency of the $m=1$ mode, normalized to the orbital frequency at the Last Stable Orbit for a Schwarzschild black hole, as a function of $\xi=r_{\text {int }} / r_{\text {LSO }}$. At large $\xi$, i.e. when the inner radius of the disk is far away from the last stable orbit, the whole disk obeys Newtonian dynamics and $\omega$ varies as $r_{\text {int }}^{-3 / 2}$. At lower $\xi$, relativistic effects modify the gas rotation curve near the inner disk edge and change the behaviour of $\omega$ : from $\xi \approx 10$ the gradient softens gradually and the the correlation is reversed at $\xi \approx 1.3$.

with a different but more elaborate method of solution; this allows us to consider more general profiles but does not change qualitatively the results presented here.

Keeping all these parameters constant, we solve the system of Eqs. (5)-(9) varying $\xi$, the ratio $r_{\text {int }} / r_{\text {LSO }}$, from 1 to 100. The results are shown in Fig. 3. As expected at large $\xi$ we recover the results obtained in the Newtonian case, since the rotation curve is unaffected by relativistic effects. In these results the mode frequency $\omega$ varies as $r_{\text {int }}^{-3 / 2}$ : since the problem has no scale length besides $r_{\text {int }}$, $\omega$ is just proportional to $\Omega_{\text {int }}$, the orbital frequency at the inner radius.

For $\xi<10$, small departures from this scaling become visible. A strong difference appears when $\xi \lesssim 1.3$ : the correlation between $\omega$ and $r_{\text {int }}$ changes sign, so that now $\omega$ decreases as $\xi$ approaches 1 .

The limited numerical resolution does not allow us to see a marked difference in the behavior of the eigenfunction (the radial dependence of the perturbed quantities) near $r_{\text {int }}$, when $\xi$ approaches 1 . Nevertheless, the values found for $\omega$ show that an ILR appears in the disk, for $\xi<1.4$ : this means that the inverse frequency-radius correlation is associated with the presence of the ILR, introduced by the relativistic effects on the rotation curve. We show in Fig. 4 the results obtained with $\beta=.5$ and $\beta=1$., normalizing this time for more clarity the mode frequency to the rotation frequency at the inner radius. At large $\xi$ the whole disk is Newtonian and $\omega / \Omega_{\text {int }}$ becomes constant. At $\beta=1$, because the frequency is higher, the ILR appears in the disk only for $\xi \simeq 1.1$, and the turnover of the frequency-inner radius correlation is also shifted to 


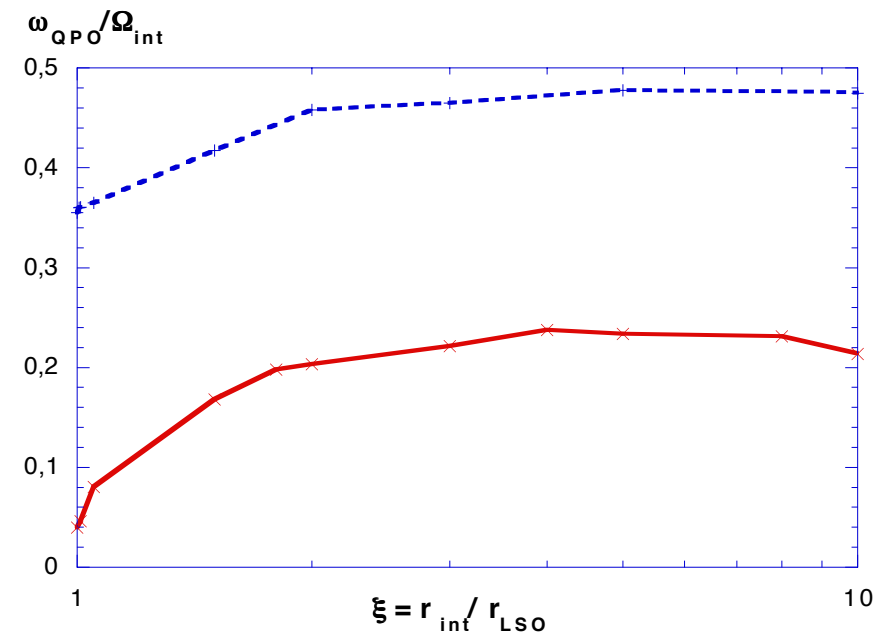

Fig. 4. Frequency of the $m=1$ mode, normalized to the orbital frequency at the inner radius, as a function of $\xi$ for $\beta=.5$ (solid) and $\beta=1$. (dashed). In the latter case the frequency is higher, so that an ILR appears in the disk only for $\xi \lesssim$ 1.1. As a result the part of the curve with a positive slope, when normalized to the last stable orbit obital frequency, is very small compared to the $\beta=0.5$ case. This motivates our presentation of the two cases normalized to the inner radius rotation frequency which allows the comparison and shows a similar behavior.

lower $\xi$. As a result the left part of the curve, with a positive correlation due to relativistic effects, shows a much more limited variation of $\omega / \Omega_{\text {int }}$.

Our main result, the change in the correlation between $\omega$ and $\xi$, is physically based on a qualitative difference in the rotation curve, which allows the mode to have an ILR in the disk when $\xi$ is close to 1 . We thus expect that this result should be quite robust and persist with more detailed relativistic models, e.g. taking into account the Kerr metrics of a spinning black hole, or more realistic profiles in the disk.

\section{The observed correlation}

As mentioned in the introduction, a QPO frequencyradius correlation, for the low-frequency QPO of blackhole binaries, has already been pointed out: in particular, for the micro-quasar GRS 1915+105, by SM97 during a particular cycle of the source, and more generally by Muno et al. who find that, among all observed properties, the QPO frequency is well correlated with the disk inner radius.

On the other hand Sobczak et al. (2000) have recently shown that two other black hole candidates, XTE J1550564 and GRO J1655-40, have contrasting behaviors: in XTE J1550-564 the QPO frequency decreases as the radius increases, as seen in GRS 1915+105, while in GRO J1655-40 the correlation is opposite. This has led us to the hypothesis that these sources lie on opposite sides of our theoretical curve, Fig. 3, i.e. in GRO J165540 the disk inner radius would be very close to the last stable orbit, while in XTE J1550-564 (and apparently in GRS 1915+105) the disk would be farther from the black hole.

In Paper I we have critically reassessed the observational results of SMR on GRO J1655-40, taking into account the uncertainties on the relation between the observed color radius and the disk inner radius; we have then turned to GRS 1915+105 because its high variability let us expect to explore the theoretical curve over a broader radial range.

\subsection{Fit parameters}

The theoretical curve uses non-dimensional parameters, namely $\xi=r_{\text {int }} / r_{\text {LSO }}$ and $\omega / \Omega\left(r_{\text {LSO }}\right)$. This will allow us to compare on the same figure objects with different masses and spins. Before showing results from the comparison between the theory and observation we will briefly present the formula and physical range for the last stable orbit radius $r_{\mathrm{LSO}}$ and the rotation frequency at this radius $\Omega\left(r_{\mathrm{LSO}}\right)$.

The general formula for the last stable orbit radius and the rotation frequency at that orbit in the Nowak \& Wagoner pseudo-Newtonian potential are

$r_{\mathrm{LSO}}=\frac{G M}{c^{2}}\left(3+A_{2} \pm \sqrt{\left(3-A_{1}\right)\left(3+A_{1}+2 A_{2}\right)}\right)$

with:

$A_{1}=1+\sqrt[3]{1-a_{\star}^{2}}\left(\sqrt[3]{1+a_{\star}}+\sqrt[3]{1-a_{\star}}\right)$

$A_{2}=\sqrt{3 a_{\star}^{2}+A_{1}^{2}}$

$a_{\star}=\frac{J}{M c}$

and:

$\Omega\left(r_{\mathrm{LSO}}\right)=\sqrt{\frac{G M}{r_{\mathrm{LSO}}^{3}}}$.

Where $J$ is the angular momentum of the black hole, and $a_{\star}$ its specific angular momentum.

These expressions can be normalized with the value for a one solar mass black hole, namely the Last Stable Orbit $r_{\bullet}=8.9 \mathrm{~km}$ and rotation frequency $\Omega_{\bullet}=1.4 \times 10^{4} \mathrm{~s}^{-1}$. We note $\delta=3+A_{2} \pm \sqrt{\left(3-A_{1}\right)\left(3+A_{1}+2 A_{2}\right)}$ and $m$ the black hole mass in units of the solar mass, giving:

$r_{\mathrm{LSO}}=\frac{\delta}{6} \quad m \quad r$

$\Omega\left(r_{\mathrm{LSO}}\right)=\left(\frac{6}{\delta}\right)^{3 / 2} \frac{1}{m} \Omega$

$\delta$ varies continously from 1 for a prograde maximally rotating Kerr black hole $\left(a_{\star}=+1\right)$ to 6 for $a_{\star}=0$, i.e. a Schwarzschild black hole and 9 for a retrograde maximally rotating Kerr black hole $\left(a_{\star}=-1\right)$.

The black hole mass gives us the range of variation for the last stable orbit radius. Table 1 shows the allowed ranges. For GRO J1655-40, we have used the value $7 M_{\odot}$, given by Orosz \& Bailyn (1997). For GRS 1915+105, we 


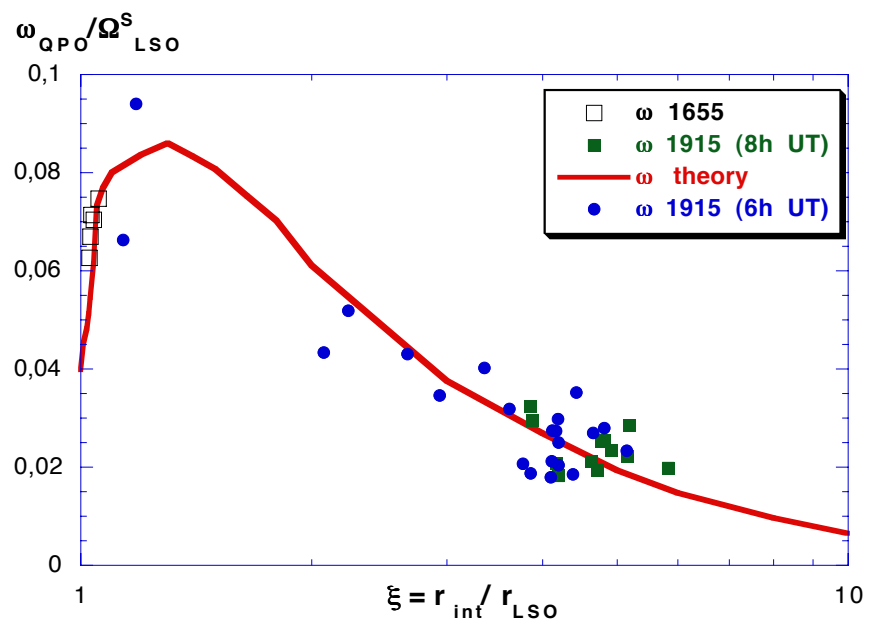

Fig. 5. Plot of the QPO frequency vs. the radius for both sources, together with the theoretical curve from TP99. Both axes as normalized in such a way that the plot is mass and spin independent. All the data points are taken from Tables 1 and 2 of Paper I.

have used the value $14 M_{\odot}$ given by Greiner et al. (2001). $a_{\star}=0.93$ is the value found by Zhang et al. (1997) for GRO J1655-40, given here as an example of almost maximally rotating black hole.

\subsection{Results from fits}

We show in Fig. 5 the observational results for both sources (retaining only data points found valid by the analysis of Paper I), fitted with the theoretical curve.

The theoretical curve is the one shown in Fig. 2 for a ratio of the gas thermal pressure to the magnetic pressure in the disk $\beta=.5$, and the same radial profiles as in TP99. Changing these parameters changes the absolute value of the frequency but not the overall behavior although, as discussed in Sect. 5.2, a value of $\beta \lesssim 1$ would not allow us to reproduce the whole range of frequency variation for GRO J1655-40.

We have fit the data points by fixing, for each source, a reference value for the radius and QPO frequency. The choice of value for these reference points are taken in the range allowed by the mass determination of the objects, see Table 1.

In this range we fit the data curves by moving them solidly up and down (i.e. fitting the fiducial value of the rotation frequency at the Last Stable Orbit), and sideways (i.e. fitting the fiducial value of $r_{\mathrm{LSO}}$ ), which changes neither their shape nor their variation with the ratio $r_{\text {int }} / r_{\text {LSO }}$. We choose to do this because the absolute relation between the observed $r_{\text {col }}$ and the real $r_{\text {int }}$ cannot be constrained, given the uncertainties in the model used for the spectral fits, and because our theoretical values correspond to a fiducial choice of disk parameters. However it is quite remarkable that, identifying the absolute values of $r_{\text {col }}$ and $r_{\text {int }}$, our best fit gives for GRO J1655-40 a spin parameter $a_{\star} \simeq .946$ for a mass of $7 M_{\odot}$, whereas from
Table 1. Range of possible last stable orbit radius for GRO J1655-40 and GRS 1915+105 depending on the rotation parameter $a_{\star}$.

\begin{tabular}{lcc}
\hline \hline spin & GRO J1655 & GRS 1915 \\
\hline$a_{\star}=1$ & 10.4 & 20.8 \\
$a_{\star}=0.93$ & 21.9 & 43.7 \\
$a_{\star}=0$ & 62.3 & 124.6 \\
$a_{\star}=-1$ & 93.4 & 186.9 \\
\hline
\end{tabular}

Table 2. Results given by the fit of the data by the theoretical curve. The spin values in braket are from Zhang et al. (1997).

\begin{tabular}{lccc}
\hline \hline Object & $r_{\text {LSO }}(\mathrm{km})$ & mass $\left(M_{\odot}\right)$ & spin \\
\hline GRO J1655 & 20.5 & 7 & $0.946(0.93)$ \\
GRS 1915 & 34.5 & 14 & $0.9765(0.998)$ \\
\hline
\end{tabular}

a different procedure Zhang et al. (1997) find $a_{\star}=.93$. For GRS $1915+105$ we obtain a spin of 0.9765 , compatible with the 0.998 found by Zhang et al. (1997). These parameters are summarized in Table 2.

This agreement between independent estimates may however be a pure coincidence, since both are model dependent: in particular we have taken a ratio $r_{\mathrm{int}} / r_{\mathrm{col}}=1$; changing this would change the determination of $r_{\mathrm{LSO}}$, and thus ultimately of the spin; Zhang et al. (1997) use a model of disk emission, as discussed in the conclusions of Paper I, and changing their hardening factor would have a similar result. Inversely Strohmayer (2001) finds a lower spin value for GRO J1655-40, based on relativistic precession models (see e.g. Stella \& Vietri 1999). The present work can thus be considered only as one more contribution in this debate, attempting to constrain the disk and fit parameters from different perspectives.

It is also noteworthy that, before the estimate of Greiner et al. (2001) for the mass of GRS 1915+105 was available, we had left this parameter free and the best fit gave us a mass of $\sim 15 M_{\odot}$, very close to the measured value, for an almost maximally (0.99965) rotating Kerr black hole. On the other hand, for GRO J1655-40 a value of $\beta \approx 1$, giving a lower inner radius for the turnover of the frequency-radius correlation, would not have allowed a satisfying fit because (as shown in Fig. 4) the theoretical range of variation of $\omega$ is too small.

Thus the agreement found from Fig. 5 would mean that in GRO J1655-40 the inner radius stays very close to the last stable orbit, whereas in GRS 1915+105 (which is in a different spectral state) it is already much larger when the QPO has appeared and the spectral fits return reliable values of $r_{\mathrm{col}}$.

The two data points for GRS $1915+105$ at $\xi \approx 1.2$ are puzzling: it would be very tempting to place them on the left (growing) part of the theoretical curve, as for GRO J1655-40. A minor change in the fit parameters would easily accomodate that. We prefer to consider this as inconclusive since the observational evidence is 
fragile: these points are the first two in this cycle of the source, and are in fact obtained before the dip (i.e. the transition to the low-hard state) as explained in Paper I. They correspond to the phase, at the end of the high state, when the inner radius starts moving away from the low value (presumably very close to $r_{\mathrm{LSO}}$ ) it has in this state. We have discussed in Paper I how these points could in fact be subject to the corrections of MFR. Furthermore, for the second (highest) of these points we have also explained that the determination of the frequency is not certain, and that it might in fact be a harmonic of the fundamental frequency.

\section{Discussion}

The Accretion-Ejection Instability appears as a good candidate to explain the low-frequency QPO of black-hole binaries, for a number of reasons: its frequency which lies in the observed range, its connection with the corona and high-energy emission, the fact that it is an instability, i.e. does not need an ad hoc excitation mechanism, and that the magnetic field configurations used for MHD models of jets obey the instability criterion. In this paper we have shown that, if one accepts this identification of the AEI as the source of the QPO, we are able to understand an otherwise unexplained behavior. The theory predicts an observational signature, the turnover of the relation between the QPO frequency and the inner radius of the disk. The relation between this radius and the color radius, extracted from spectral fits, is still too uncertain to give more than an indicative value to the fits we present. It is however very comforting that these fits, which also depend on assumptions on the physical parameters in the disk, give results in surprisingly close agreement with independent estimates. More observations showing the changing correlations (perhaps even in a single source at different times) would be needed to confirm it. These observations could extend to neutron-star binaries, since Psaltis et al. (1999a) find a link between the low-frequency QPOs in many different X-ray binaries. Future work will be dedicated to this, and to testing the suggestion we made in Paper I that the anomalous color radius frequently found in spectral fits of different sources (including the two studied here) might indicate the presence of a spiral shock or hot point in the disk.

\section{References}

Balbus, S. A., \& Hawley, J. F. 1991, ApJ, 376, 214

Binney, J., \& Tremaine, S. 1987, Galactic Dynamics (Princeton University Press)

Blandford, R. D., \& Znajek, R. L. 1977, MNRAS, 179, 433B

Casse, F., \& Ferreira, J. 2000, A\&A, 353, 1115

Caunt, S., \& Tagger, M. 2001, A\&A, 367, 1095

Chandrasekhar, S., 1960, Proc. Nat. Acad. Sci., 46, 253

Dhawan, V., Mirabel, I. F., \& Rodriguez, L. F., ApJ, 543, 373

Greiner, J., Cuby, J. G., McCaughren, M. J. 2001, Nature, 414, 522

Markwardt, C. B., Swank, J. H., \& Taam, R. E., 1999, ApJ, 513,37

Merloni, A., Fabian, A. C., \& Ross, R. R. 2000, MNRAS, 313, 193 (MFR)

Miller, M. C., Lamb, F. K., \& Psaltis, D. 1998, ApJ, 508, 791

Muno, M. P., Morgan, E. H., \& Remillard, R. A. 1999, ApJ, 52,321

Nowak, M. A., \& Wagoner, R. V. 1992, ApJ, 393, 607

Nowak, M. A. 2000, MNRAS, 318, 361

Orosz, J. A., \& Bailyn, C. D. 1997, ApJ, 482, 1086

Paczynski, B., \& Witta, P. J. 1980, A\&A, 88, 23

Papaloizou, J. C. B., \& Pringle, J. E. 1985, MNRAS, 213, 799

Psaltis, D., Belloni, T., \& van Der Klis, M. 1999a, ApJ, 520, 262

Psaltis, D., Wijnands, R., Homan, J., et al. 1999b, ApJ, 520, 763

Psaltis, D., \& Norman, C. 2002, ApJ, submitted [astro-ph/0001391]

Psaltis, D. 2002, ApJ, submitted [astro-ph/0010316]

Rodriguez, J., Varnière, P., Tagger, M., \& Durouchoux, P. 2002, A\&A, 387, 487 (Paper I)

Shimura, T., \& Takahara, F. 1995, ApJ, 445, 780

Sobczak, G. J., McClintock, J. E., Remillard, R. A., et al. 2000, ApJ, 531, 537, (SMR)

Stella, L., \& Vietri, M. 1999, ApJ, 524, L63

Strohmayer, T. E. 2001, ApJ, 552, L49

Swank, J., Chen, X., Markwardt, C., \& Taam, R. 1997, Proc. of the Conf. Accretion Processes in Astrophysics: Some Like it Hot, held at U. Md., October 1997, ed. S. Holt, \& T. Kallman

Tagger, M. 1999, Proc. of the 5th Compton Symp., Portshmouth (USA), AIP Conf. Proc., 510, 129 [astro-ph/9910365]

Tagger, M., Henriksen, R. N., Sygnet, J. F. \& Pellat, R. 1990, ApJ, 353, 654

Tagger, M., \& Pellat, R. 1999, A\&A, 349, 1003 (TP99)

Varnière, P., \& Tagger, M. 2002, A\&A, submitted

Velikhov, E. P. 1959, Sov. Phys. JETP Lett., 9, 995

Zhang, S. N., Cui, W., \& Chen, W. 1997, ApJ, 482, L155 